\title{
Comparison of RPL GD-301 and TLD-100 detectors responses by Monte Carlo simulation
}

\author{
A-H. Benali ${ }^{1,2}$, G. Medkour Ishak-Boushaki ${ }^{2}$, A. Nourreddine $^{3}$, M. Allab ${ }^{2}$ \\ ${ }^{1}$ Faculty of Science of Nature and Life, Univ. Echahid Hamma Lakhdar, El-oued Algeria. \\ ${ }^{2}$ Laboratory SNIRM-Faculty of Phys., Univ. of Sciences and Technology Houari Boumediène, Algiers Algeria. \\ ${ }^{3}$ Institut Pluridisciplinaire Hubert Curien de Strasbourg, France.
}

\begin{abstract}
LiF:Mg,Ti) Thermo Luminescent Detectors are widely used for monitoring patient dose in radiotherapy treatments whereas Radio-Photoluminescent Dosimeters (RPL) are increasingly devoted to radiological protection purposes. A study, aiming at extending the use of RPL glasses to clinical applications, is conducted by comparing the dosimetric characteristics of a RPL glass dosimeter, commercially known as GD-301 to those of a TLD -100 detector. In this paper, preliminary Monte Carlo simulation results describing these dosimeters responses in terms of absorbed dose, sourcedetector distance and characteristics of the incident gamma field are presented.
\end{abstract}

\section{Introduction}

A radiation dosimeter is a detector used to measure or evaluate, either directly or indirectly, quantities required for radiation protection purposes as the exposition rate, kerma, absorbed dose or equivalent dose.

For radiation protection applications, two kinds of dosimeters are used: active or operational dosimeter and passive one. The first device measures absorbed dose in real time. The second gives integrated absorbed dose over a period of time [1].

Some materials, known as luminescent detectors, when irradiated emit a quantity of light proportional to the absorbed ionizing radiation. Three groups of luminescence detectors are applied in personal dosimetry: thermoluminescence detectors (TLDs), detectors based on optically stimulated luminescence (OSLDs) and radiophotoluminescence (RPL) glasses [2, 3].

Thermoluminescent dosimeters (TLDs) are frequently used for monitoring ambient and personnel doses. The readout process of these devices needs a heat treatment. The great disadvantage of these dosimeters is the fact that their readout cannot be repeated: luminescents centers, created by ionizing radiation, disappear after heating [4].

Radiophotoluminescent glass dosimeters (RPLGDs) have the advantage to be read repeatedly, because the readout process does not eliminate the luminescent centers [5].

Actually, RPLGD are increasingly used for monitoring ambient dose and personnel dose but are not yet regularized for monitoring patients dose in radiotherapy treatments.

In view to evaluate the use of RPLGD in vivo dosimetry [6], we have undertaken a comparative study, by Monte Carlo simulation, between the dosimetric characteristics of a RPL glass dosimeter, commercially known as GD-301 and those of a TLD -100 detector. We give here the preliminary results.

\section{Dosimetry system}

For our study, a model GD-301 glass dosimeter (AGC Techno Glass Corp., Shizuoka, Japan) is used. The model GD-301 is $1.5 \mathrm{~mm}$ in diameter and $8.5 \mathrm{~mm}$ in length. Weight composition of the glass dosimeter was as follows: $31.55 \% \mathrm{P}, 51.16 \% \mathrm{O}, 6.12 \% \mathrm{Al}, 11.00 \% \mathrm{Na}$, and $0.17 \% \mathrm{Ag}$. Effective atomic number and density of the glass dosimeter were 12.039 and $2.61 \mathrm{~g} / \mathrm{cm}^{3}$, respectively [6].

The TLD-100 dosimeter is made of lithium fluoride (LiF) crystals in the form of chips $\left(3 \times 3 \times 1 \mathrm{~mm}^{3}\right.$ in dimensions) with density of $2.635 \mathrm{~g} / \mathrm{cm}^{3}$. Because its effective atomic number of 8.3 close to that of water or tissue, LiF-TLD is used routinely for dose measurements in radiotherapy. The used TLD-100 contains $26.7 \%$ Lithium, 73.2\% Fluorine, $200 \mathrm{ppm}$ Magnesium, and approximately $10 \mathrm{ppm}$ of Titanium. This type of TLD100 is denoted as TLD-A [4, 7].

The dosimeters are irradiated in a depth of $10 \mathrm{~cm}$ of a solid water phantom. The phantom is a parallelepiped with dimensions of $30 \times 30 \times 30 \mathrm{~cm}^{3}$ and weight composition as follows: $8.09 \% \mathrm{H}, 67.17 \% \mathrm{C}, 2.42 \% \mathrm{~N}$, $19.87 \% \mathrm{O}, 0.13 \% \mathrm{Cl}, 2.32 \% \mathrm{Ca}$ and $0.17 \% \mathrm{Ag}$. Effective atomic number and density of the solid water phantom were 3.57 and $1.015 \mathrm{~g} / \mathrm{cm}^{3}$, respectively [4].

\section{Monte Carlo simulation}

Monte Carlo N-Particle Transport Code System [8] was 
used to calculate the absorbed radiation dose in each luminescent detector.

The experimental set up modeled by MCNP5 code consists in a punctual photon point source collimated in a cone of rectangular extremity surface equal to the $\mathrm{X}$ ray field sizes used in radiotherapy treatments. The photon cone irradiates a luminescent dosimeter placed in a depth of $10 \mathrm{~cm}$ from the surface of the solid water phantom. Figure 1 gives the modeled experimental set-up used for Monte Carlo simulation.

The "F6 tally" for photons was used to record the energy deposition in each studied dosimeter. For ensure all statistical checks recommended by MCNP5 code, a total of 700 millions starting photons were considered per simulation run.

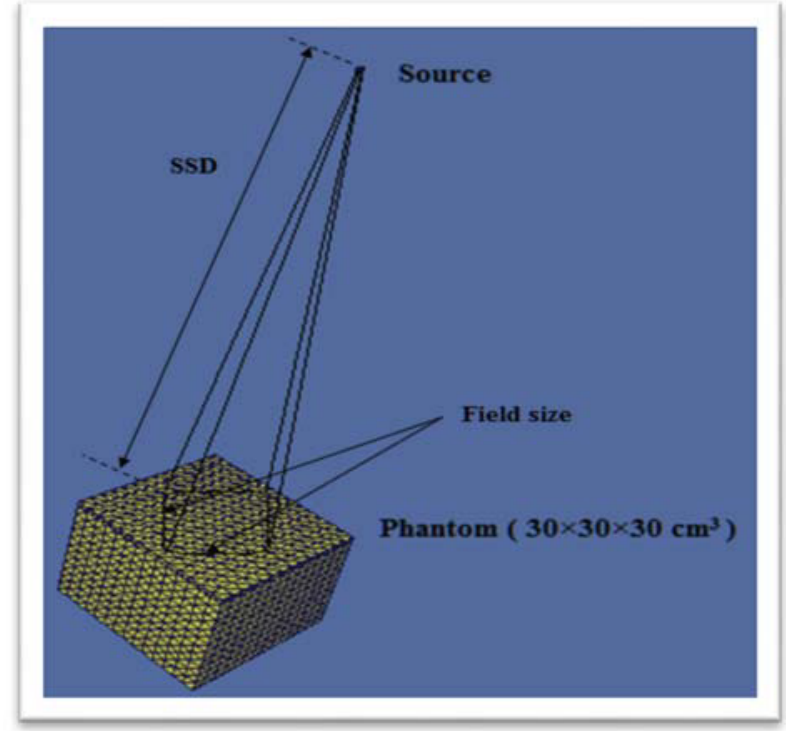

Figure 1. Experimental set-up modeled

\section{Dosimetric characteristics}

Aiming to use RPL detectors in vivo dosimetry, Monte Carlo simulation of some dosimetric characteristics, for both TLD-100 and RPL GD 301 detectors, have been carried out. We give here preliminary simulation results.

\subsection{SSD dependence}

The effect of the distance source-detector (SSD) on dose measurements was investigated by considering the experimental set-up of Fig. 1. For Monte Carlo simulation, we have considered a punctual source emitting photons of $3.6 \mathrm{MeV}$ energy (corresponding to the mean energy of $15 \mathrm{MV}$ X-ray beams) in a cone of $10 \times 10 \mathrm{~cm}^{2}$ area at the solid water phantom superficies (corresponding to a fixed field size of $10 \times 10 \mathrm{~cm}^{2}$ ). The dose absorbed, at $10 \mathrm{~cm}$ depth in the solid water phantom, was calculated when increasing the SSD from 85 to $115 \mathrm{~cm}$. The results of simulation, for both dosimeters RPL GD-301 and TLD-100 are reported in Fig. 2. The calculated doses are normalized to the dose value evaluated at SSD of $100 \mathrm{~cm}$. A good agreement is achieved between the two dosimeters responses except at $115 \mathrm{~cm}$ where a discrepancy of about $1 \%$ is noticed.

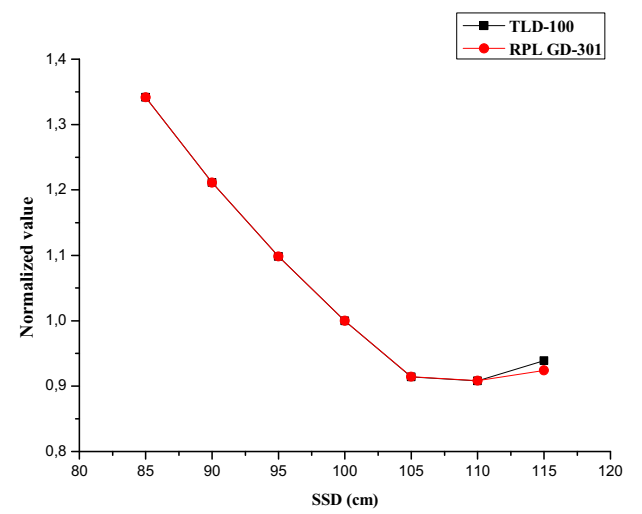

Figure 2. SSD dependence for glass and TLD dosimeters.

\subsection{Field size dependence}

For the same geometry of Fig. 1, the absorbed energy in each investigated dosimeter, irradiated by a $15 \mathrm{MV}$ Xray beams at a distance of $100 \mathrm{~cm}$, evaluated by Monte Carlo simulation and carried out for different field sizes varying from $5 \times 5 \mathrm{~cm}^{2}$ to $20 \times 20 \mathrm{~cm}^{2}$ are reported in Fig. 3. The calculated absorbed energies values are normalized to the value estimated for a reference field size of $10 \times 10 \mathrm{~cm}^{2}$. A negligible discrepancy between the results of the RPL GD-301 and TLD-100 dosimeter is noticed.

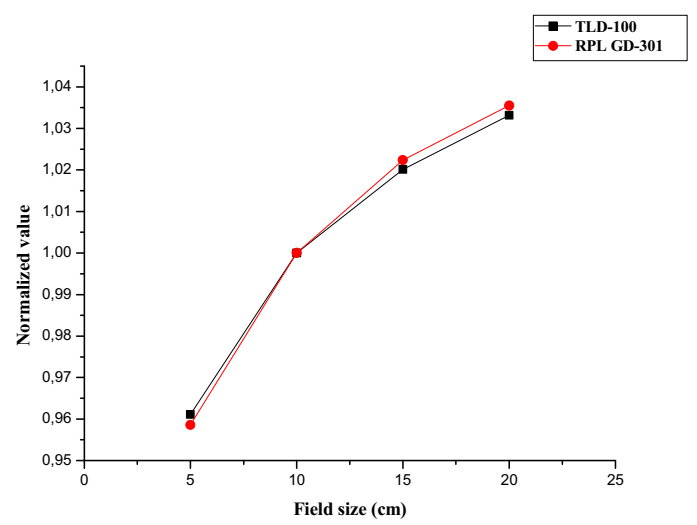

Figure 3. Field size dependence of the absorbed energy in RPLGD-301 and TLD-100 dosimeter.

\section{Conclusion}

This paper is a preliminary comparative study, by Monte Carlo simulation using MCNP5 code, of dosimetry characteristics between a Radio photoluminescent and a thermoluminescent dosimeter.

The aim of this work is to evaluate the use of an RPL dosimeter known commercially as RPLGD-301, instead of a TLD-100 dosimeter, to monitor patient's doses in radiotherapy treatments.

The first Monte Carlo simulation results show a very similar behavior of the variation of the absorbed dose in 
either RPL or TLD dosimeter versus the source detector distance and the irradiated field size. This work is in progress.

\section{References}

1. E.B. Podgorsak. External Photon Beams: Physical Aspects. In: E.B. Podgorsak, Radiation oncology physics: A Handbook for teachers and students, Vienna, Austria: IAEA 2005, 71-100. ISBN 92-0107304-6.

2. Pawe Olko, Radiation Measurements 45 (2010) 506511.

3. Z. Knezevi, et al., Radiation Measurements 57 (2013) 918.

4. D Baltas, L Sakelliou, N Zamboglou, The Physics of Modern Brachytherapy for Oncology, New York, USA: Taylor \& Francis Group, 2007.

5. S.-M. Hsu, et al., Radiation Measurements 43 (2008) $538-541$.

6. J.-E. Rah, U.-J. Hwang, H. Jeong, S.Y. Park, Radiation Measurements 2011; 46: 40-45.

7. H. Asni, et al., Journal of Engineering Thermophysics, 2011; Vol. 20, No. 3: 329-333.

8. MCNP-A General Monte Carlo N-Particle Transport Code, Version 5 Los Alamos National Laboratory 2003. 